\title{
A multicentre retrospective cohort study on COVID-19-related physical interventions and adult hospital admissions for ENT infections
}

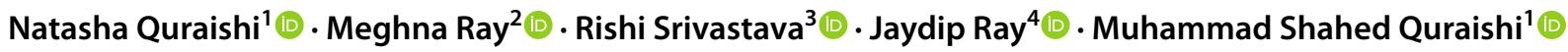

Received: 28 September 2021 / Accepted: 6 November 2021 / Published online: 22 November 2021

(c) Crown 2021

\begin{abstract}
Purpose To report changes in adult hospital admission rates for acute ENT infections following the introduction of COVID19-related physical interventions such as hand washing, use of face masks and social distancing of 2-m in the United Kingdom.

Methods Retrospective cohort study comparing adult admissions with acute tonsillitis, peritonsillar abscess, epiglottitis, glandular fever, peri-orbital cellulitis, acute otitis media, acute mastoiditis, retropharyngeal abscess and parapharyngeal abscess in the 1-year period after the introduction of COVID-related physical interventions (2020-2021) with a 1-year period before this (2019-2020) in three UK secondary care ENT departments.

Results In total, there were significantly fewer admissions for ENT infections ( $n=1073,57.56 \%, p<0.001$; RR 2.36, 95\% CI $[2.17,2.56])$ in the 2020-2021 period than in the 2019-2020 period. There were significant reductions in admissions for tonsillitis $(64.4 \% ; p<0.001)$, peritonsillar abscess $(60.68 \% ; p<0.001)$, epiglottitis $(66.67 \% ; p<0.001)$, glandular fever $(38.79 \% ; p=0.001)$, acute otitis media $(26.85 \% ; p=0.01)$ and retropharyngeal and/or parapharyngeal abscesses $(45.45 \%$; $p=0.04)$.

Conclusion Our study demonstrates a sizeable reduction in adult admissions for ENT infections since the introduction of COVID-19-related physical interventions. There is evidence to support the use of physical interventions in the prevention of viral transmission of respiratory disease. Preventing ENT infections requiring admission through simple physical interventions could be of great benefit to the quality of life of patients and economical benefit to healthcare systems.
\end{abstract}

Keywords COVID-19 $\cdot$ Social distancing $\cdot$ Masks $\cdot$ Hand hygiene $\cdot$ ENT $\cdot$ Infections

\section{Introduction}

On $23^{\text {rd }}$ March 2020, the United Kingdom (UK) entered a national lockdown due to the global COVID-19 pandemic. The Department of Health and Social Care implemented the 'Hands. Face. Space.' campaign [1]. This multimedia campaign advised physical interventions (PIs) to improve public hygiene such as washing hands for $20 \mathrm{~s}$, wearing face masks and social distancing of 2-m and to limit all unnecessary travel. Viral respiratory infections such as COVID-19 spread

Muhammad Shahed Quraishi

Muhamad.quraishi@nhs.net

Doncaster Royal Infirmary, Doncaster, UK

University of Leicester, Leicester, UK

Nottingham University Hospitals, Nottingham, UK

4 Sheffield Teaching Hospitals, Sheffield, UK via droplet and aerosol transmission with global pandemics being driven by high levels of transmission in an environment of heavy viral load amongst susceptible populations [2-4].

There is increasing evidence to support the use of PIs such as social distancing, hand washing and face masks to slow the spread of respiratory viruses such as Covid-19 [5-7]. A recent Cochrane review suggested the benefits of mask wearing was uncertain but hand washing and social distancing may be beneficial. However, it noted limitations including low-moderate certainty of evidence and high risk of bias in the trials that were included [2]. PIs appear to be most effective when combined with one another, rather than utilised in isolation [8].

In addition to reducing rates of COVID-19, there appears to have been a concurrent reduction in other respiratory conditions. Vasquez-Hoyos and colleagues noted a significant reduction $(83 \%)$ in respiratory admissions in paediatric 
intensive care during the COVID-19 pandemic compared with the pre-COVID-19 period [9].

\section{Objective}

We aim to report changes in adult hospital admission rates for acute ENT infections following the introduction of COVID-19-related PIs.

\section{Methods}

The STROBE reporting guideline was used in the preparation of this manuscript [10].

\section{Ethical considerations}

The data and analysis were part of service evaluations and so NHS Research Ethics Committee review is not required according to the NHS Research Authority [11].

\section{Study design and setting}

A retrospective cohort study was undertaken utilising hospital data obtained from three large National Health Service (NHS) hospitals (1 district general hospital and 2 tertiary centres) covering a catchment population of over 2 million in central England, UK. Adult hospital admissions for ENT infections over a 12-month period (March 2019 to February

Table 1 NHS codes for conditions of interest

\begin{tabular}{ll}
\hline NHS code(s) & ENT infections \\
\hline J030, J038, J039 & Acute tonsillitis \\
J36X & Peritonsillar abscess \\
J051 & Epiglottitis \\
B279 & Glandular fever \\
H050 & Peri-orbital cellulitis \\
H669 & Acute otitis media \\
H700 & Acute mastoiditis \\
J390 & Retropharyngeal \\
& abscess/parapharyn- \\
& geal abscess \\
\hline
\end{tabular}

2020 inclusive) before the COVID-19 pandemic were compared with a 12-month period from 23 March 2020 (when the UK government implemented the first national lockdown) to March 2021 inclusive. The data were retrieved by hospital information analysts using standard NHS Codes for eight common ENT infectious conditions (Table 1). No identifiable patient data were accessed.

\section{Participants}

Patients aged 16 and older admitted to hospital for acute tonsillitis, peritonsillar abscess, epiglottitis, glandular fever, peri-orbital cellulitis, acute otitis media, acute mastoiditis, retropharyngeal abscess and parapharyngeal abscess were included.

\section{Main outcome measures}

The main outcome measures were the number of adult hospital admissions.

\section{Statistical analysis}

Statistical analysis was undertaken by a qualified statistician (AO) using the Open Epi website [12]. Statistical significance was calculated using the $\chi^{2}$ test with significance defined as $p<0.05$. Relative risk (RR) and respective $95 \%$ Confidence Intervals (CI) were also calculated.

\section{Results}

Across all centres there was a total of 1864 adult admissions in the 2019-2020 period and 791 adult admissions in 2020-2021 period for the ENT infections of interest. The cumulative average age was 32 years (16-97 years) in the 2019-2020 period and 35 years (17-99 years) in the 2020-2021 period (see Table 2 for results for each centre).

In total, there were significantly fewer total admissions in the 2020-2021 period than in the 2019-2020 period $(n=1073,57.56 \%, p<0.001 ; \mathrm{RR} 2.36,95 \%$ CI $[2.17,2.56])$ with there being reductions in all ENT infections studied (see Tables 3, 4).

Table 2 Number of adult admissions and ages across each centre for each time period

\begin{tabular}{|c|c|c|c|c|c|c|c|c|}
\hline & \multicolumn{2}{|l|}{ A } & \multicolumn{2}{|l|}{ B } & \multicolumn{2}{|l|}{$\mathrm{C}$} & \multicolumn{2}{|l|}{ Total } \\
\hline & 2019-2020 & 2020-2021 & 2019-2020 & 2020-2021 & 2019-2020 & 2020-2021 & 2019-2020 & 2020-2021 \\
\hline Admission & 550 & 235 & 265 & 157 & 1049 & 399 & 1864 & 791 \\
\hline Average age (years) & 33 & 36 & 30.49 & 31.75 & 32.42 & 36.18 & 32 & 35 \\
\hline Age range (years) & $17-97$ & $17-96$ & $17-78$ & $17-81$ & $16-94$ & $16-99$ & $16-97$ & $17-99$ \\
\hline
\end{tabular}




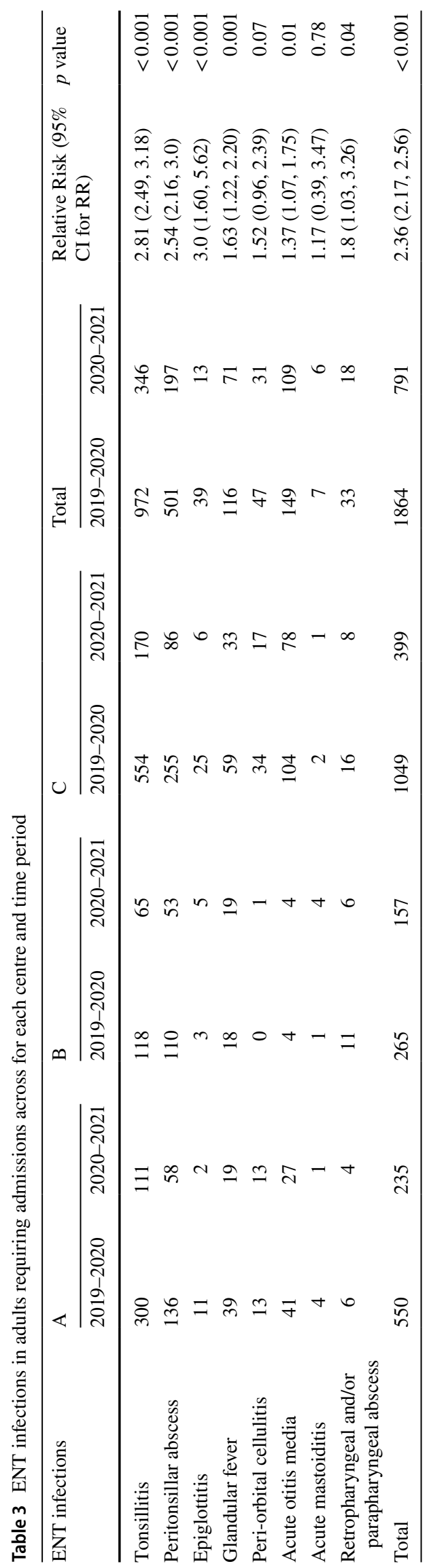

In both years, the majority of ENT infections requiring admissions were tonsillitis and peritonsillar abscess (Fig. 1).

\section{Tonsillitis}

There was a significant total reduction of 626 admissions (64.4\%) for tonsillitis in the 2020-2021 period compared with the 2019-2020 period ( $p<0.001$; RR $2.81,95 \%$ CI $[2.49,3.18])$, with every centre having fewer admissions for tonsillitis in the 2020-2021 period (Fig. 2a).

\section{Peritonsillar abscess}

There was a significant total reduction of 304 admissions $(60.68 \%)$ for peritonsillar abscess in the 2020-2021 period compared with the 2019-2020 period significant $(p<0.001$; RR $2.81,95 \%$ CI $[2.49,3.18])$, with every centre having fewer admissions for peritonsillar abscesses in the 2020-2021 (Fig. 2b).

\section{Epiglottitis}

There was a significant total reduction of 26 admissions (66.67\%) for epiglottitis in the 2020-2021 period compared with the 2019-2020 period ( $p<0.001$; RR 3.0, 95\% CI $[1.60,5.62])$. Two centres, $\mathrm{A}$ and $\mathrm{C}$, showed a reduction in admissions for epiglottitis (A: 9 (81.82\%); C: 19 (76\%)), whereas $\mathrm{B}$ showed an increase in epiglottitis requiring admission (2(66.67\%)) (Fig. 2c).

\section{Glandular fever}

There was a significant total reduction of 45 admissions (38.70\%) for glandular fever in the 2020-2021 period compared with the 2019-2020 period ( $p=0.001$; RR 1.63, 95\% CI $[1.22,2.20])$. Two centres, $\mathrm{A}$ and $\mathrm{C}$, showed a reduction in admissions for glandular fever (A: 20 (51.28\%); C: 26 $(44.07 \%))$, whereas B showed an increase in glandular fever requiring admission (1 (5.56\%)) (Fig. 2d).

\section{Peri-orbital cellulitis}

There was a total reduction of 16 admissions (34.04\%) for peri-orbital cellulitis in the 2020-2021 period compared with the 2019-2020 period, however, this was not significant $(p=0.07$; RR 1.52, 95\% CI [0.96, 2.39]). Results varied from centre to centre with no change in $\mathrm{A}$, an increase of 1 at B and a decrease of 17 (50\%) at C (Fig. 2e).

\section{Acute otitis media}

There was a significant total reduction of 40 admissions (26.85\%) for acute otitis media in the 2020-2021 period 
Table 4 Change in admissions for ENT conditions in 2020-2021 period compared with 2019-2020 period

\begin{tabular}{|c|c|c|c|c|c|c|c|c|}
\hline \multirow[t]{2}{*}{ Change in Admissions } & \multicolumn{2}{|l|}{ A } & \multicolumn{2}{|l|}{$\mathrm{B}$} & \multicolumn{2}{|l|}{$\mathrm{C}$} & \multicolumn{2}{|l|}{ Total } \\
\hline & Number & Percentage & Number & Percentage & Number & Percentage & Number & Percentage \\
\hline Tonsillitis & -189 & $-63.00 \%$ & -53 & $-44.92 \%$ & -384 & $-69.31 \%$ & -626 & $-64.40 \%$ \\
\hline Peritonsillar abscess & -78 & $-57.35 \%$ & -57 & $-51.82 \%$ & -169 & $-66.27 \%$ & -304 & $-60.68 \%$ \\
\hline Epiglottitis & -9 & $-81.82 \%$ & 2 & $66.67 \%$ & -19 & $-76.00 \%$ & -26 & $-66.67 \%$ \\
\hline Glandular fever & -20 & $-51.28 \%$ & 1 & $5.56 \%$ & -26 & $-44.07 \%$ & -45 & $-38.79 \%$ \\
\hline Peri-orbital cellulitis & 0 & $0.00 \%$ & 1 & N/A & -17 & $-50.00 \%$ & -16 & $-34.04 \%$ \\
\hline Acute otitis media & -14 & $-34.15 \%$ & 0 & $0.00 \%$ & -26 & $-25.00 \%$ & -40 & $-26.85 \%$ \\
\hline Acute mastoiditis & -3 & $-75.00 \%$ & 3 & $300.00 \%$ & -1 & $-50.00 \%$ & -1 & $-14.29 \%$ \\
\hline $\begin{array}{l}\text { Retropharyngeal and/or } \\
\text { parapharyngeal abscess }\end{array}$ & -2 & $-33.33 \%$ & -5 & $-45.45 \%$ & -8 & $-50.00 \%$ & -15 & $-45.45 \%$ \\
\hline Total & -315 & $-57.27 \%$ & -108 & $-40.75 \%$ & -650 & $-61.96 \%$ & -1073 & $-57.56 \%$ \\
\hline
\end{tabular}

(a) $2019-20$

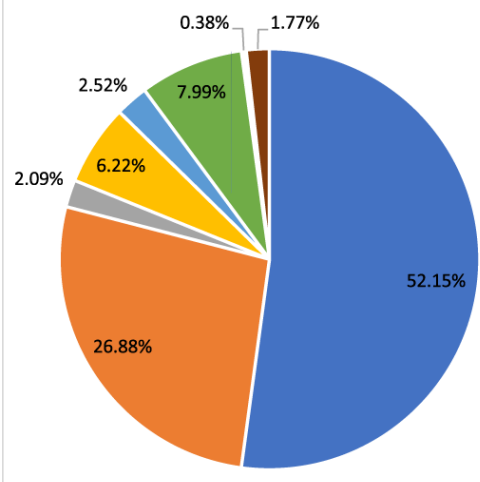

- Tonsillitis

- Peritonsillar abscess

- Epiglottitis

- Glandular fever

- Periorbital cellulitis

- Acute otitis media

Acute mastoiditis

- Retropharyngeal and/or parapharyngeal abscess

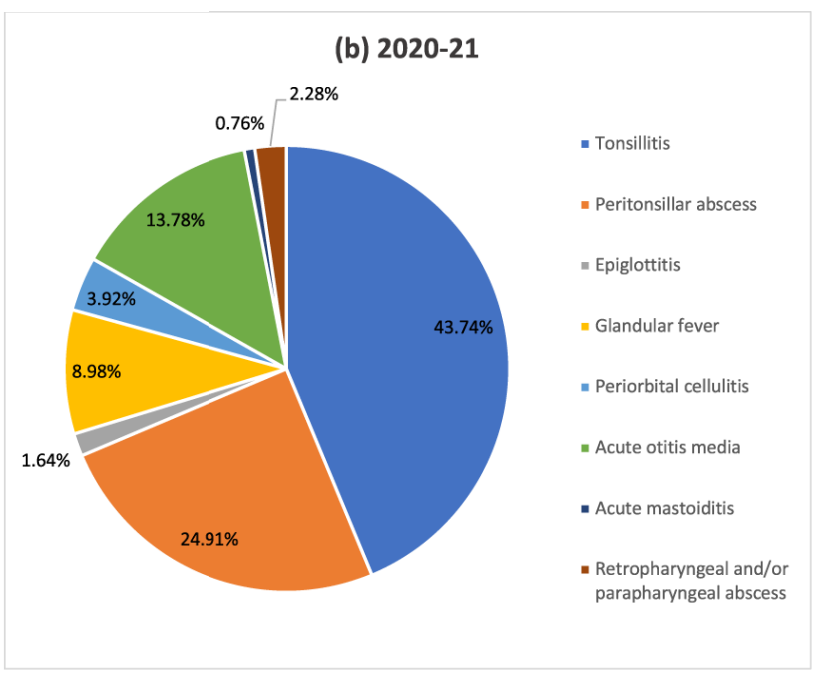

Fig. 1 The proportion of adult admissions by infection in a 2019-2020 and b 2020-2021

compared with the 2019-2020 period ( $p=0.01$; RR 1.37, $95 \% \mathrm{CI}[1.07,1.75])$. Two centres, $\mathrm{A}$ and $\mathrm{C}$, showed a reduction in admissions for acute otitis media (A: 14 (34.15\%); C: $26(25 \%))$, whereas B showed no change acute otitis media requiring admission (Fig. $2 \mathrm{f}$ ).

\section{Acute mastoiditis}

There was a total reduction of 1 admission (14.29\%) for acute mastoiditis in the 2020-2021 period compared with the 2019-2020 period ( $p=0.78$; RR 1.17, 955 CI [0.39, 3.47]). Two centres, $\mathrm{A}$ and $\mathrm{C}$, showed a reduction in admissions for acute mastoiditis (A: 3 (75\%); C: 1 (50\%)), whereas $\mathrm{B}$ showed an increase in acute mastoiditis requiring admission (3 (300\%)) (Fig. 2g).

\section{Retropharyngeal and/or parapharyngeal abscess}

There was a significant total reduction of 15 admissions $(45.45 \%)$ for retropharyngeal and/or parapharyngeal abscess in the 2020-2021 period compared with the 2019-2020 period ( $p=0.04$; RR 1.8, 95\% CI [1.03, 3.26]). Every centre had fewer admissions for retropharyngeal and/or parapharyngeal abscess in the 2020-2021 (Fig. 2h).

\section{Discussion}

Our review of three centres has shown a significant reduction in adult admissions for ENT infections of $57.56 \%$ $(p<0.001)$. Most notably, there have been significant reductions in admissions for tonsillitis $(64.4 \% ; p<0.001)$, peritonsillar abscess $(60.68 \% ; p<0.001)$, epiglottitis 


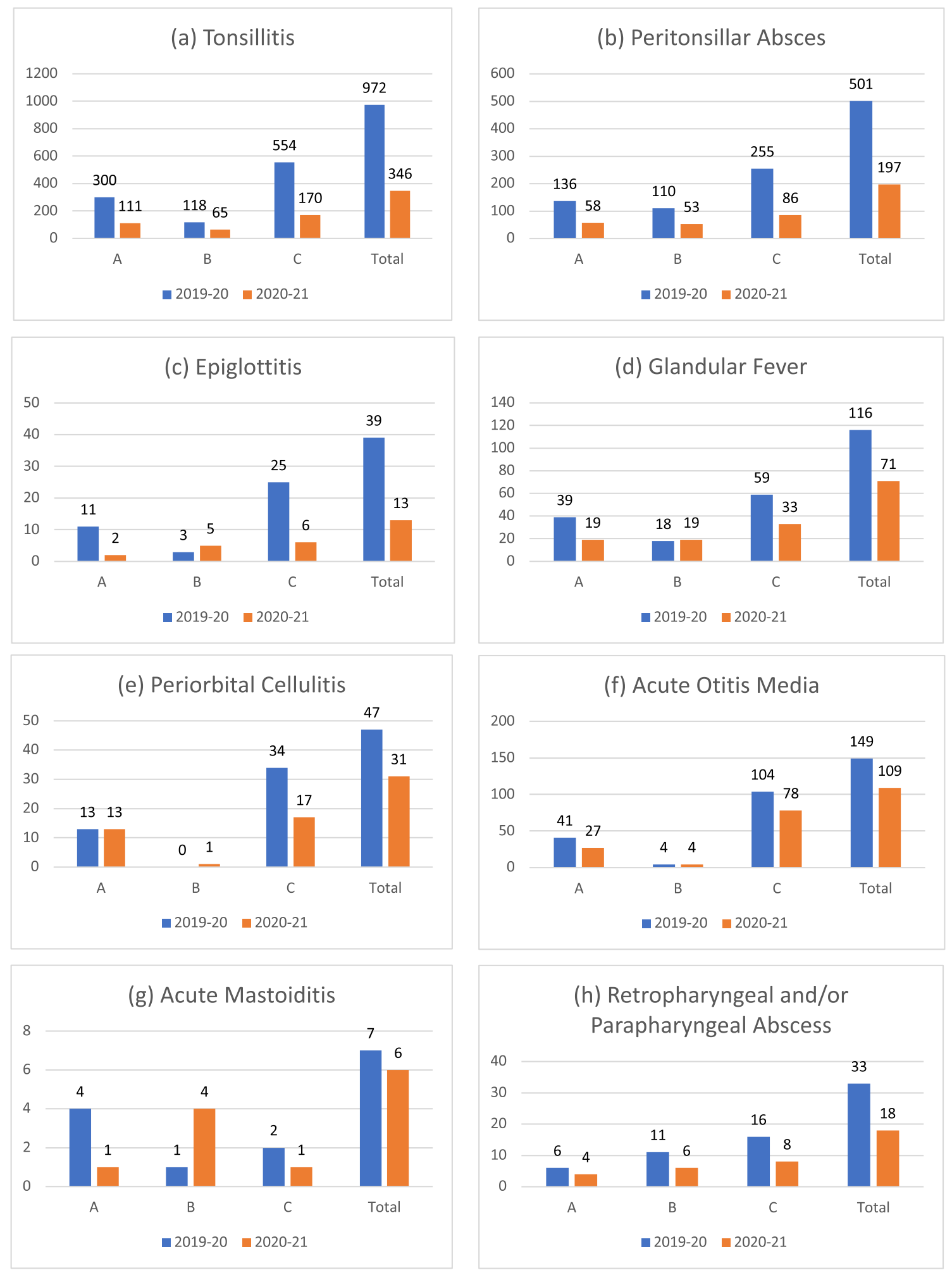

Fig. 2 Admissions by centre and in total for a tonsillitis, $\mathbf{b}$ peritonsillar abscess, $\mathbf{c}$ epiglottitis, $\mathbf{d}$ glandular fever, e peri-orbital cellulitis, $\mathbf{f}$ acute otitis media, $\mathbf{g}$ acute mastoiditis and $\mathbf{h}$ retropharyngeal abscess and/or parapharyngeal abscess 
(66.67\%; $p<0.001)$, glandular fever $(38.79 \% ; p=0.001)$, acute otitis media $(26.85 \%$; $p=0.01)$ and retropharyngeal and/or parapharyngeal abscesses $(45.45 \% ; p=0.04)$.

\section{COVID-19 physical interventions and ENT infections}

Our study appears to be the only large multicentre study in English literature focussing purely on adult admissions with ENT infections but there is limited data from other smaller local studies on other topics which had similar findings.

The centres included, covered catchment areas of varying sizes and populations and so this is reflected in the numbers of admissions observed. The figures for B reflect that as a centre it only covers ENT admissions within its own Trust. Both A and C admit ENT patients from their own and other hospitals within the region such as Rotherham and Worksop in the case of A and Barnsley and Chesterfield in the case of $\mathrm{C}$.

Smith [13] is one of the few papers that considers hospital admissions. They conducted a 12-week prospective multicentre national audit on admission avoidance in tonsillitis and peritonsillar abscess in the UK. They noted 344 admissions for tonsillitis and 226 admissions for peritonsillar abscess over their study period. However, they did not obtain comparative data from pre-COVID-19. They compared their rate of two peritonsillar abscesses per centre per month during the study period with the higher rate of 3.9 peritonsillar abscesses per centre per month measured from the UK Multicentre Audit of Quinsies (MAQ) in 2016. They attributed this reduction to PIs. While our rate of 5.5 peritonsillar abscesses per centre per month during the 2020-2021 period is higher than that of MAQ (2016), it is markedly lower than our rate of 13.9 peritonsillar abscesses per centre per month in the pre-COVID 2019-20 period. Stansfield et al. [14] undertook a retrospective review study on ENT emergencies over two comparable 3-month periods, pre- and post-COVID-19. They found a statistically significant reduction in admissions for tonsillar infection overall $(p<0.005)$ post-COVID.

Our findings are supported by a reduction in ENT infection in other clinical settings, all of which have been considered related to PIs. A cross-sectional single centre retrospective study, assessing the number of episodes of tonsillitis in children awaiting a tonsillectomy, found a significant reduction in the frequency of tonsillitis during the lockdown period compared with before lockdown [15]. In the USA, the Centre for Disease Control (CDC) reported that ED visits due to specific upper respiratory tract infections, including middle ear infections (otitis media), were amongst those showing largest declines in presentation when compared with a pre-pandemic period [16]. A retrospective study comparing Otolaryngology admissions in a University Hospital in Jordan, from the lockdown period to the same dates in the year 2019, found a reduction in cases of head and neck infections [17].

Campagnoli and colleagues (2021) found reductions in the number of ED consultations for otologic issues (mostly infections) $(-88.5 \%, p<0.01)$ and tonsillitis $(-87.5 \%$, $p=0.688$ ) during the pandemic compared with the preceding year [18]. Another retrospective study from three centres in Italy also noted a reduction in emergency intervention for infectious diseases which was attributed to PIs [19].

McBride and colleagues [20] in the USA observed a significant reduction in the incidence of streptococcal pharyngitis, acute otitis media, and infectious mononucleosis per 100,000-person years during the closure of schools due to COVID-19 with a $\mathrm{RR}=0.36(p<0.0001), \mathrm{RR}=0.60$ $(p<0.0001)$, and $\mathrm{RR}=0.84(p=0.0117)$. Van de Pol and colleagues (2021) performed a retrospective study comparing primary care patients in March 2020-May 2020 $(405,688)$ and March 2019-May 2019 (389,708). They noted a decline in antibiotic prescriptions rate for respiratory/ear infections from $21 \%$ to $13 \%$ ( $-8 \%$ (CI, $-8.8 \%$ to $-7.2 \%)$ ), while other infectious diseases remained similar or slightly increased, which they felt was explained by PIs [21].

\section{Strengths and limitations}

The literature has frequently cited reluctance to attend ED due to fears regarding COVID-19 as a possible reason for a reduction in attending ED and primary care. However, in our study, we have focussed on adult patients who have been reviewed and considered to require admission. We would suggest that even if patients may have initially been reluctant to attend hospital during the COVID-19 period, those that became unwell enough to require admission would likely still attend. Therefore, a strength of our study is that we focussed on admissions in comparison to other studies.

A point to consider is that the three centres included are in different counties within the UK. Therefore, there may have been some slight variation in lockdown rules over the 12-month COVID-19 period. However, we are focussing more on physical intervention measures relating to hand washing, wearing of face masks and social distancing. These are the basis of the "Hands. Face. Space." Campaign which remained throughout the country despite these local changes. We should also consider that during the initial stages of the COVID-19 pandemic guidance was issued aiming to reduce hospital admissions and the implementation of this will have varied between centres.

\section{Consideration for future public health}

Strict implementation of PIs, as demonstrated in China, has shown reduction in COVID-19 infection rates. Whilst ethical issues surrounding such drastic combined measures are 
relatively complex, the healthcare, societal and economic benefits of reduced infection cannot be overemphasised [22].

In the pre-COVID era, a significant portion of acute clinical practice in Otolaryngology involved managing ENT infections. It was unknown if COVID-19-related PIs would affect the incidence of non-COVID upper respiratory tract infections such as ENT infections. Our study demonstrates a reduction in adult admissions for ENT infections following the introduction of such measures. These results were not completely unexpected but the scale of reduction has been much larger than expected, especially the reduction in admissions for tonsillitis and peritonsillar abscess which accounted for the majority of adult admissions for ENT infections (79.03 and 68.65\% in 2019-2020 and 2020-2021, respectively).

This reduction may have important implications on resource savings for healthcare systems such as the NHS and on public health. In our clinical experience, the average adult admitted with an ENT infection requires 2 days of inpatient care and may require up to 1 week off work post-discharge. The observed reduction in adult admissions equates to 2146 bed-days saved for the three centres during the 12 months of PIs. Similarly, this reduction would benefit general public health with less time off work and a better quality of life. With a UK population of approximately 68 million, an estimated 72,964 bed-days would be saved nationally each year based on our findings. While there may be some variation in the incidence of ENT infections in different regions of the UK due to other factors such as weather, demographics and social conditions, the projected resource-savings for the healthcare systems could be considerable.

Previously, use of face masks or avoidance of handshakes were limited to certain populations that had previously experienced the effects of airborne transmission during SARS and MERS outbreaks. Our results suggest a large benefit in continuing PIs even after COVID-19.

\section{Conclusion}

Our study demonstrates a sizeable reduction in adult admissions for ENT infections since the introduction of PIs (hand washing, mask wearing and social distancing) due to the COVID-19 pandemic. There is evidence to support the use of PIs in the prevention of viral transmission of respiratory disease. Preventing ENT infections requiring admission through simple PIs could be of great benefit to the quality of life of patients and economical benefit to healthcare systems.

Acknowledgements Special thanks to Dr. Aamir Omair (Coordinator, Research Unit, College of Medicine, King Saud bin Abdulaziz University for Health Sciences) for his help with the statistical analysis
Author contributions NQ made substantial contributions to the conception and design of the work; the acquisition, analysis, or interpretation of data; drafted the work and revised it critically for important intellectual content; approved the version to be published; and agrees to be accountable for all aspects of the work in ensuring that questions related to the accuracy or integrity of any part of the work are appropriately investigated and resolved. MR made substantial contributions to the conception and design of the work; approved the version to be published; and agrees to be accountable for all aspects of the work in ensuring that questions. RS made substantial contributions to the acquisition, analysis, or interpretation of data; drafted the work and revised it critically for important intellectual content; approved the version to be published; and agrees to be accountable for all aspects of the work in ensuring that questions related to the accuracy or integrity of any part of the work are appropriately investigated and resolved. JR made substantial contributions to the acquisition, analysis, or interpretation of data; drafted the work and revised it critically for important intellectual content; approved the version to be published; and agrees to be accountable for all aspects of the work in ensuring that questions related to the accuracy or integrity of any part of the work are appropriately investigated and resolved. MSQ made substantial contributions to the conception and design of the work; the acquisition, analysis, or interpretation of data; drafted the work and revised it critically for important intellectual content; approved the version to be published; and agrees to be accountable for all aspects of the work in ensuring that questions related to the accuracy or integrity of any part of the work are appropriately investigated and resolved.

Funding None to declare.

\section{Declarations}

Conflict of interest The authors have no relevant financial or non-financial interests to disclose. The authors have no conflicts of interest to declare that are relevant to the content of this article. All authors certify that they have no affiliations with or involvement in any organization or entity with any financial interest or non-financial interest in the subject matter or materials discussed in this manuscript. The authors have no financial or proprietary interests in any material discussed in this article.

Availability of data and materials Research data and materials are not shared.

Code availability Not applicable.

Ethics approval The data and analysis were part of service evaluations and so NHS Research Ethics Committee review is not required according to the NHS Research Authority.

Consent to participate Not applicable.

Consent for publication Not applicable.

\section{References}

1. Care DoHaS. Press Release: New campaign to prevent spread of coronavirus indoors this winter. https://www.gov.uk/government/ news/new-campaign-to-prevent-spread-of-coronavirus-indoorsthis-winter: UK Government; 2020 [Web page on UK government website for press release of Department of Health and Social's 
new campaign. The 'Hands. Face. Space' public information campaign urges the public to continue to wash their hands, cover their face and make space to control infection rates and avoid a second peak.]. https://www.gov.uk/government/news/new-campaign-toprevent-spread-of-coronavirus-indoors-this-winter

2. Jefferson T, Del Mar CB, Dooley L, Ferroni E, Al-Ansary LA, Bawazeer GA et al (2020) Physical interventions to interrupt or reduce the spread of respiratory viruses. Cochrane Database Syst Rev 11:CD006207

3. Herfst S, Böhringer M, Karo B, Lawrence P, Lewis NS, Mina MJ et al (2017) Drivers of airborne human-to-human pathogen transmission. Curr Opin Virol 22:22-29

4. Kutter JS, Spronken MI, Fraaij PL, Fouchier RA, Herfst S (2018) Transmission routes of respiratory viruses among humans. Curr Opin Virol 28:142-151

5. Leung NHL, Chu DKW, Shiu EYC, Chan KH, McDevitt JJ, Hau BJP et al (2020) Respiratory virus shedding in exhaled breath and efficacy of face masks. Nat Med 26(5):676-680

6. Cowling BJ, Leung GM (2020) Epidemiological research priorities for public health control of the ongoing global novel coronavirus (2019-nCoV) outbreak. Euro Surveill 25(6):2000110

7. Ferris M, Ferris R, Workman C, O'Connor E, Enoch DA, Goldesgeyme E, et al. FFP3 respirators protect healthcare workers against infection with SARS-CoV-2. 2021. Authorea [Internet]. 4 July 2021. https://doi.org/10.22541/au.162454911.17263721/v2

8. Regmi K, Lwin CM (2021) Factors associated with the implementation of non-pharmaceutical interventions for reducing coronavirus disease 2019 (COVID-19): a systematic review. Int J Environ Res Public Health 18(8):4274

9. Vásquez-Hoyos P, Diaz-Rubio F, Monteverde-Fernandez N, Jaramillo-Bustamante JC, Carvajal C, Serra A et al (2020) Reduced PICU respiratory admissions during COVID-19. Arch Dis Child. https://doi.org/10.1136/archdischild-2020-320469

10. Group SI. STROBE Statement https://www.strobe-statement. org/fileadmin/Strobe/uploads/checklists/STROBE_checklist_v4_ cohort.pdf: STROBE Initiative Group; 2007. https://www.strobestatement.org/fileadmin/Strobe/uploads/checklists/STROBE_ checklist_v4_cohort.pdf

11. Authority NHR. Defining Research Table http://www.hra-decis iontools.org.uk/research/docs/DefiningResearchTable_Oct2017-1. pdf: NHS Health Research Authority; 2017. http://www.hra-decis iontools.org.uk/research/docs/DefiningResearchTable_Oct2017-1. pdf

12. Dean AG SK, Soe MM. OpenEpi: Open Source Epidemiologic Statistics for Public Health, Version www.OpenEpi.com2013
13. Smith ME (2020) Admission avoidance in tonsillitis and peritonsillar abscess: a prospective national audit during the initial peak of the COVID-19 pandemic. Clin Otolaryngol. https://doi.org/10. 1111/coa. 13680

14. Stansfield J, Dobbs S, Harrison R, Lee K, Sharma S, Okour K et al (2021) Management of ENT emergencies during the coronavirus disease 2019 pandemic. J Laryngol Otol 135(2):117-124

15. Heward E, Rocke J, Kumar N, Izzat S (2020) Recurrent tonsillitis and parental perceptions of tonsillectomy during the COVID-19 pandemic. Int J Pediatr Otorhinolaryngol 139:110463

16. Hartnett KP, Kite-Powell A, DeVies J, Coletta MA, Boehmer TK, Adjemian J et al (2020) Impact of the COVID-19 pandemic on emergency department visits-United States, January 1, 2019May 30, 2020. MMWR 69(23):699-704

17. Al Omari A, Kanaan Y, Nuseir A, Al-Ashqar R, Al-Balas H, Hamarneh O et al (2021) The impact of COVID-19 outbreak on otolaryngology practice, jordanian experience: a qualitative study. Ann Med Surg (Lond) 62:435-439

18 Campagnoli M, Cerasuolo M, Renna M, Dell'Era V, Valletti PA, Garzaro M (2021) ENT referral from emergency department during COVID-19: a single-center experience. Ear Nose Throat J. https://doi.org/10.1177/0145561320984560

19. Pontillo V, Iannuzzi L, Petrone P, Sciancalepore PI, D’Auria C, Rinaldi M et al (2020) ENT surgical emergencies during the COVID-19 outbreak. Acta Otorhinolaryngol Ital 40(6):399-404

20. McBride JA, Eickhoff J, Wald ER (2020) Impact of COVID-19 quarantine and school cancelation on other common infectious diseases. Pediatr Infect Dis J 39(12):e449-e452

21. van de Pol AC, Boeijen JA, Venekamp RP, Platteel T, Damoiseaux RAMJ, Kortekaas MF et al (2021) Impact of the COVID-19 pandemic on antibiotic prescribing for common infections in The Netherlands: a primary care-based observational cohort study. Antibiotics (Basel) 10(2):591

22. Fernstrom A, Goldblatt M (2013) Aerobiology and its role in the transmission of infectious diseases. J Pathog. https://doi.org/10. $1155 / 2013 / 493960$

Publisher's Note Springer Nature remains neutral with regard to jurisdictional claims in published maps and institutional affiliations. 